\title{
Energy transfer in a static electric field
}

\author{
David L. Andrews and Alexander M. Bittner \\ School of Chemical Sciences, University of East Anglia, Norwich, UK.
}

Received 4 November 1992

Revised 30 March 1993

Accepted 30 March 1993

\begin{abstract}
The issue of how the application of a static electric field can influence energy transfer between molecules is addressed. Several aspects are considered. First, in molecules of reasonably high symmetry where the donor decay or the acceptor excitation transition is electric dipole forbidden, the application of a static field can, by an electro-optical interaction, allow energy to transfer by a dipolar mechanism. In this way application of the field effectively switches on the transfer process. Secondly, it is shown how the application of a static electric field can provide more spectroscopic information, as in the technique of electric field-induced spectroscopy: it also offers a possible basis for new types of laser system. Finally, in a polar liquid an applied field can produce a degree of molecular alignment. It is demonstrated how this can significantly modify energy transfer characteristics, and the dependence of the effect on field strength and temperature are identified.
\end{abstract}

\section{Introduction}

One of the principal mechanisms by means of which electronically excited molecules become deactivated is through the transfer of their energy to other molecules [1]. Molecular energy transfer plays a highly significant role in a wide range of materials, and in recent years particular attention has been paid to its role in the solid state and in biological systems. In the former, much interest currently accrues to the study of energy transfer in crystalline media such as those involved in lasers and ancillary devices. Where living organisms are concerned the main areas of current research relate to intermolecular energy migration in photosynthetic systems, and intramolecular transfer in protein structures, where measurement of the process constitutes an important means of determining the distances between subunits.

Correspondence to: Dr. D.L. Andrews, School of Chemical Sciences, University of East Anglia, Norwich NR4 7TJ, UK. Telefax: + 44-603 259396 .
The theory of molecular energy transfer distinguishes between several mechanisms; the most important are electron exchange coupling and the Coulombic coupling of transition moments [2]. The first type is restricted to neighbouring molecules with overlapping wave functions (see for example ref. [3]). The latter type of transfer couples molecules over a considerable range of distances and is more frequently observed; here the transition moments of the species (atoms or molecules) involved are commonly both electric dipoles (as for example in ref. [4]), but cases involving one electric dipole and one quadrupole [5] or two electric quadrupoles [6] are observed, too. The familiar Förster theory deals with the most common case of two electric dipole-allowed molecular transitions for molecules at distances $R \leqslant \hbar c / \Delta E$, (the so-called near zone), where $\Delta E$ is the energy transferred; this leads to an energy transfer rate that is inversely proportional to the sixth power of $R$ [1]. A more recent theoretical approach includes the effect of molecules at larger intermolecular distances by employing molecular quantum electrodynamics. Here the coupling between the 
transition dipole moments is formulated in terms of virtual photons which travel between the participating molecules. Radiative energy transfer is thereby automatically accounted for, too [7,8], and the inclusion of higher electric and also magnetic multipole moments is straightforward [9].

It is the purpose of this paper to address the issue of how the application of a static electric field can influence energy transfer between molecules, and to develop a theory applicable to a wide range of systems. There are several aspects to be considered. First, it is possible with molecules of reasonably high symmetry for the donor decay or the acceptor excitation transition to be electric dipole forbidden. Although higher multipole transition moments might still mediate the transfer process, they are generally associated with relatively weak coupling. In such cases the application of a static field can, by an electro-optical interaction, allow energy to transfer by a dipolar mechanism. In this way application of the field effectively switches on the transfer process. Secondly, the application of a static electric field can provide more spectroscopic information: the technique of electric-fieldinduced spectroscopy invokes two-quantum selection rules for one-quantum absorption (an example is given in ref. [10]) and the quantum electrodynamical theory is well developed $[11,12]$. Thirdly, in a polar liquid an applied field can produce a degree of molecular alignment, significantly modifying energy transfer characteristics. This temperature-dependent effect has been discussed for electric-field-induced photoabsorption [12] and it is important for energy transfer processes, too. Moreover the characteristic dependence of the effect on field strength and on temperature are both readily identifiable.

\section{Quantum electrodynamical formulation}

The aim is to derive rate equations for the transfer of energy between a donor species A and an acceptor $\mathrm{B}$,

$\mathrm{A}^{*}+\mathrm{B} \rightarrow \mathrm{A}+\mathrm{B}^{*}$.

Here the initial state of $A$, to be labelled $|\alpha\rangle$, is in general an energy level belonging to the manifold of an electronic excited state. Molecule B initially resides in one of the sub-levels of the ground electronic state, usually the $S_{0}$ state and therefore arbitrarily labelled $|0\rangle$. The process of energy transfer entails a downward transition in A to a sub-level usually belonging to the ground electronic state, again labelled $|0\rangle$, accompanied by an upward transition by molecule $B$ to a state labelled $|\beta\rangle$ : use of the designation $|0\rangle$ for the lower energy levels is not meant to signify any kind of degeneracy, and the theory to be developed below in no way depends on these states necessarily being ground states. The overall process is clearly governed by the energy conservation requirement

$E_{x 0}=E_{p 0}$,

where $E_{\alpha 0}$ and $E_{\beta 0}$ respectively denote the energy differences between the excited states of $A$ and $B$ and their lower states. To develop the theoretical formulation in detail, it is first necessary to introduce the quantum electrodynamics which will properly accommodate the features of interest.

Molecular quantum electrodynamics is distinctive in its application of quantum theory not only to the molecules but also to radiation fields [13]. The modes of the radiation field are in the present application occupied only by transverse virtual photons as in the Power-Zienau-Woolley formulation [14]: the photons are called virtual because they are not observable, but mediate the electromagnetic coupling between participating molecules. For completeness, and also in order to introduce a number of parameters which are employed subsequently, it is appropriate first to consider the common case in which both the donor decay and the acceptor excitation transitions are electric dipole allowed, and for which the rate of energy transfer in the absence of any static field remains significant.

\subsection{Dipole-allowed energy transfer}

The overall Hamiltonian $H$ for the donor acceptor system can be separated as:

$H=H_{\text {mol }}+H_{\text {rad }}+H_{\text {int }}$. 


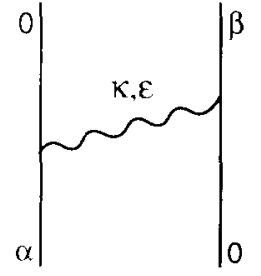

A

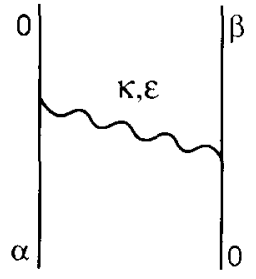

A (a)

(b)

Fig. 1. The two time-ordered diagrams for energy transfer $A^{*}+B \rightarrow A+B^{*}$. The wavy line denotes a virtual photon of wave vector $\kappa$ and polarisation $\varepsilon$.

The term $H_{\text {mol }}$ is a sum of the molecular Hamiltonians for the two molecules and $H_{\text {rad }}$ pertains to the radiation field. Coupling between the molecules and the radiation field is described by the perturbing Hamiltonian

$H_{\text {int }}=-\varepsilon_{0}^{-1}\left[\boldsymbol{\mu}_{\mathrm{A}} \cdot \boldsymbol{d}^{\perp}\left(\boldsymbol{R}_{\mathrm{A}}\right)+\boldsymbol{\mu}_{\mathrm{B}} \cdot \boldsymbol{d}^{\perp}\left(\boldsymbol{R}_{\mathrm{B}}\right)\right]$.

The symbols $\mu_{\mathrm{A}}$ and $\mu_{\mathrm{B}}$ here denote the electric dipole moment operators of $\mathrm{A}$ and $\mathrm{B}$, and the position vectors of the molecules are $\boldsymbol{R}_{\mathrm{A}}$ and $\boldsymbol{R}_{\mathrm{B}}$, respectively. The transverse electric displacement operator is given by

$$
\begin{aligned}
d^{\perp}(\boldsymbol{r})= & i \sum_{\boldsymbol{\kappa}, \varepsilon}\left(\varepsilon_{0} h c \kappa / 2 V\right)^{1 / 2} \\
& \times\left[\varepsilon a \exp (\mathrm{i} \boldsymbol{\kappa} \cdot \boldsymbol{r})-\bar{\varepsilon} a^{+} \exp (-\mathrm{i} \boldsymbol{\kappa} \cdot \boldsymbol{r})\right],
\end{aligned}
$$

where $V$ stands for the quantisation volume, $\boldsymbol{\kappa}$ is the virtual photon wave vector and $\varepsilon$ its polarisation vector, and $a, a^{+}$denote the corresponding annihilation and creation operators, respectively.

In the absence of any applied field the energy transfer process can be represented by two timeordered diagrams (see fig. 1). By summing the corresponding contributions by the usual methods $[7,13]$ it is found that the pertaining transition matrix element can be written as

$M^{\mathrm{fi}}=\mu_{i}^{\alpha 0} \mu_{j}^{\beta 0} V_{(i j)}(K, R)$,

using the Einstein summation convention for repeated tensor indices (as usual, and throughout this paper, specific axial indices $x, y$ and $z$ are exempt from the sum rule). The virtual photon coupling tensor $V_{(i j)}$ is defined by

$$
\begin{aligned}
V_{(i j)}(K, R)= & \left(4 \pi \varepsilon_{0} R^{3}\right)^{-1} \exp (\mathrm{i} K R) \\
& \times\left[\delta_{i j}-\hat{R}_{i} \hat{R}_{j}\right)(1-\mathrm{i} K R) \\
& \left.-\left(\delta_{i j}-\hat{R}_{i} \hat{R}_{j}\right) K^{2} R^{2}\right],
\end{aligned}
$$

where

$$
\begin{aligned}
& \boldsymbol{R}=\boldsymbol{R}_{\mathrm{B}}-\boldsymbol{R}_{\mathrm{A}}, \\
& K=E_{\alpha 0} / h c=E_{\beta 0} / \hbar c
\end{aligned}
$$

and the brackets around the indices (ij) serve as a reminder of their inherent permutational symmetry. The rate of energy transfer, $\Gamma$, can now be calculated from the Fermi Golden Rule:

$$
\begin{aligned}
\Gamma & =\left(2 \pi \rho_{\mathrm{f}} / \hbar\right)\left|M^{\mathrm{fi}}\right|^{2} \\
& \left.=\left(2 \pi \rho_{\mathrm{f}} / \hbar\right) \mu_{i}^{\alpha 0} \mu_{j}^{\beta 0} \bar{\mu}_{k}^{\alpha 0} \bar{\mu}\right\}^{\beta 0} V_{(i j)}(K, \boldsymbol{R}) \bar{V}_{(k l)}(K, \boldsymbol{R}) .
\end{aligned}
$$

The parameter $\rho_{\mathrm{f}}$ denotes the density of the final molecular states and can be expressed in terms of the individual ${ }^{\mathrm{A}} \rho_{\mathrm{f}}$ and ${ }^{\mathrm{B}} \rho_{\mathrm{f}}$ :

$\rho_{\mathrm{f}}=\int^{\mathrm{A}} \rho_{\mathrm{f}}\left(E_{\alpha 0}-E\right){ }^{\mathbf{B}} \rho_{\mathrm{f}}(E) \mathrm{d} E$.

In the near zone where $K R \ll 1$, the rate given by eq. (11) exhibits the familiar $R^{-6}$ dependence, as predicted by the classical Förster theory and as experimentally confirmed $[1,2]$. Of the additional $R^{-2}$ and $R^{-4}$ terms which arise, and which become effective at longer intermolecular distances, the former can be identified with radiative energy transfer. The latter is a distinctive retardation feature whose presence only the quantum electrodynamical theory reveals. Possible regimes for its experimental detection have recently been suggested $[8,15]$. It is also worth noting that the $R^{-2}$ radiative term gives rise to a paradox which can only be resolved if reabsorption by the sample is taken into account [8].

\subsection{Electric field-induced energy transfer}

The effects of a static electric field are incorporated into the theory by inclusion of an additional 


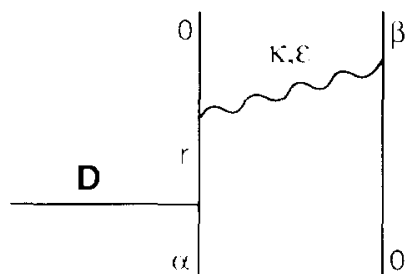

A

(a)

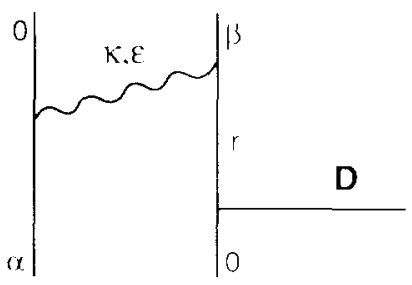

A

(b)

Fig. 2. Two of the twelve time-ordered diagrams for electric-field-induced energy transfer.

perturbational term in the Hamiltonian for the system. This term is given by

$$
H_{\mathrm{int}}^{\prime}=-\varepsilon_{0}^{-1} \boldsymbol{\mu}_{\mathrm{A}} \cdot \boldsymbol{D}-\varepsilon_{0}^{-1} \boldsymbol{\mu}_{\mathrm{B}} \cdot \boldsymbol{D},
$$

where $\boldsymbol{D}$ denotes the electric displacement vector of the static field whose magnitude is $\left(D / \varepsilon_{0}\right)$. A static electric field applied between two parallel electrodes is spatially homogeneous and of constant magnitude. Its gradient is thus zero, and the term (13) is not the first part of a multipolar series. One way forward would be to treat $H_{\text {int }}^{\prime}$ as a perturbation on the molecular Hamiltonian $H_{\text {mol }}$, leading to perturbed molecular states which would be employed as basis states for a calculation following the procedure of section 2.1. However, a different approach is taken here, namely to treat $H_{\text {int }}^{\prime}$ directly as a further perturbation on $H$; the overall Hamiltonian is now

$H^{\prime}=H_{\mathrm{mol}}+H_{\mathrm{rad}}+H_{\mathrm{int}}+H_{\mathrm{int}}^{\prime}$.

The advantage of this method, which naturally leads to the same final results, lies in the preservation of the molecular entities described by $H_{\text {mol }}$. Moreover, calculation of the transfer rate is very simple: in quantum electrodynamics a static field can be envisaged as the zero-frequency limit of a (real) photon [11]. Because the field shows no time dependence, the appropriate symbol in the time-ordered diagrams is a horizontal line. Two typical time-ordered diagrams for electric-fieldinduced energy transfer are shown in fig. 2. Altogether there are twelve such diagrams, and they show the same mirror image property discussed in refs. [9,16]: the electric field can act on either A or B.

Calculation of the transition matrix element now proceeds along similar lines to refs. [9,17] and gives

$$
\begin{aligned}
M^{\mathrm{fi}}= & -\varepsilon_{0}{ }^{1} D_{i} S_{i j}^{\gamma 0} \mu_{k}^{\beta 0} V_{(j k)}(K, \boldsymbol{R}) \\
& -\varepsilon_{0}{ }^{1} D_{i} S_{i j}^{\beta 0} \mu_{k}^{\chi 0} V_{(j k)}(K, \boldsymbol{R}),
\end{aligned}
$$

where

$S_{i j}^{\alpha 0} \equiv \sum_{r}\left[{ }^{\mathrm{A}} \mu_{i}^{\alpha r \mathrm{~A}} \mu_{j}^{r 0}\left(E_{x r}\right)^{-1}+{ }^{\mathrm{A}} \mu_{i}^{r \mathrm{OA}} \mu_{j}^{\alpha r}\left(E_{0 r}\right)^{-1}\right]$.

In passing it may be noted that the tensor $S_{i j}^{x 0}$ is identical to the molecular response tensor which features in the theory of electric field-induced absorption [11]: the tensor $S_{i j}^{\beta 0}$ is defined similarly. The energy transfer rate is again calculated with the aid of eq. (10). Using $\hat{D}$ to denote the unit vector of the static electric field, and the symbol $(\mathrm{A} \leftrightarrow \mathrm{B})$ to signify an exchange of the molecular labelling, we have:

$$
\begin{aligned}
\Gamma= & \left(2 \pi \rho_{\mathrm{f}} / \hbar\right)\left(D / \varepsilon_{0}\right)^{2} \\
& \times\left\{\hat{D}_{i} S_{i j}^{\alpha 0} \mu_{k}^{\beta 0} V_{(j k)}(K, \boldsymbol{R}) \hat{D}_{l} \bar{S}_{l m}^{\alpha 0} \bar{\mu}_{n}^{\beta 0} \bar{V}_{(m n)}(K, \boldsymbol{R})\right. \\
& +(A \leftrightarrow B)+2 \operatorname{Re}\left[\bar{D}_{i} S_{i j}^{\alpha 0} \mu_{k}^{\beta 0} V_{(j k)}(K, \boldsymbol{R})\right. \\
& \left.\left.\times \hat{D}_{l} \bar{S}_{l m}^{\beta 0} \bar{\mu}_{n}^{\alpha 0} \bar{V}_{(m n)}(K, \boldsymbol{R})\right]\right\} .
\end{aligned}
$$

The three different terms in eq. (17) result from the squared modulus of $M^{\mathrm{fi}}$, and are called the first and second diagonal and the interference term, respectively. The first term is associated with the six time-ordered diagrams in which $D$ acts on $A$; the second results from the six diagrams in which 


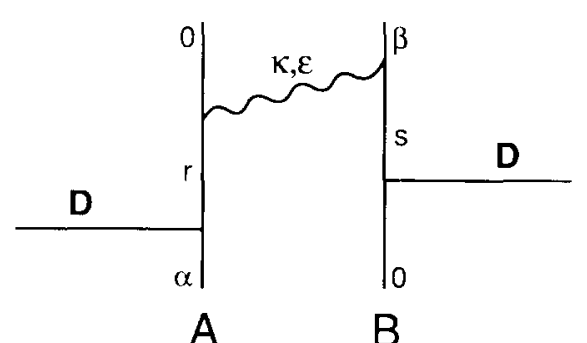

Fig. 3. Typical time-ordered diagram for doubly electric-fieldinduced energy transfer.

$D$ acts on molecule B. The third is a cross-term. Note that all parameters in eq. (17) except $V_{(i j)}$ are real-valued. The rate depends on the square of the electric field, as in electric-field-induced spectroscopy.

Using eqs. (6) and (15), we can write down a general expression for the matrix element which accommodates both field-free and field-induced terms:

$$
\begin{aligned}
M_{\mathrm{full}}^{\mathrm{fi}}= & \mu_{i}^{\alpha 0} \mu_{j}^{\beta 0} V_{(i j)}(K, \boldsymbol{R}) \\
& -\varepsilon_{0}^{-1} D_{i}\left(S_{i j}^{\alpha 0} \mu_{k}^{\beta 0}+S_{i j}^{\beta 0} \mu_{k}^{\alpha 0}\right) V_{(j k)}(K, \boldsymbol{R}) \\
& +\varepsilon_{0}^{-2} D_{i} D_{j} S_{i k}^{\alpha 0} S_{j l}^{\beta 0} V_{(k l)}(K, \boldsymbol{R})-\ldots
\end{aligned}
$$

The last term corresponds to a doubly electricfield-induced energy transfer which is depicted in fig. 3. Its contribution would require a doublequantum transition on both molecules and could therefore only be associated with a very weak effect; for this reason it is henceforth excluded from consideration. It is worth noting that if both transitions $\alpha \rightarrow 0$ and $0 \rightarrow \beta$ are dipole-allowed, then under normal circumstances the first term of eq. (18) will greatly exceed the second. Hence in order to observe electric-field-induced energy transfer (or avoid competition with normal energy transfer) one transition should be dipole-forbidden and twoquantum allowed. This leaves the second term in eq. (18) as the principal contribution and leads to the rate given by eq. (17). The rare case of a dipole and two-quantum forbidden transition (e.g. $A_{1 g} \leftrightarrow B_{1 g}$ in a $D_{6 h}$ molecule) would give rise to further terms; however, we shall restrict consideration to dipole-forbidden transitions that are twoquantum allowed.

\section{Rotationally averaged energy transfer rates}

As they stand, eqs. (11) and (17) can only directly be applied to molecules with fixed mutual orientation. Moreover eq. (17) is only applicable when the static field operates in a fixed direction with respect to each pair, e.g. for a crystalline sample. If the molecules are in a fluid phase, a rotational average has to be performed between the laboratory coordinates to which $D$ is referred and the molecular coordinates to which A, B and $\boldsymbol{R}$ are referred [18]. The number of tensor indices involved is called the rank of the average. This procedure gives the rotating pair rate (denoted by angular brackets) which applies for any two molecules in a fixed mutual orientation in a fluid (e.g. two chromophores within a large molecule in solution). For free molecules two further averages are required in order to decouple both molecular coordinate frames from $\boldsymbol{R}$ [19]. For free molecules these latter averages are also required in eq. (11) for the field-free rate. Other scenarios are possible, e.g. a single average is required if $\mathrm{A}$ denotes an atom or a freely rotating molecule in a crystal, or two averages if this condition applies to both $A$ and B. To develop the theory further, however, we restrict the scope to rotating pairs and free molecules and consider in more detail the various possibilities which arise.

\subsection{Non-polar molecules}

Consider first the case where $\mathrm{A}$ and $\mathrm{B}$ have permanent electric dipole moments $\mu^{00}$ which are both either zero or negligibly small, in the sense that $\left(\boldsymbol{\mu}^{00} \cdot \boldsymbol{D} / \varepsilon_{0}\right) / k_{\mathrm{B}} T \ll 1$, where $k_{\mathrm{B}}$ is the Boltzmann constant and $T$ the temperature of the fluid. In such circumstances field-induced orientation in any fluid sample can be ignored, the molecular orientational distribution being isotropic.

\subsubsection{Dipole-allowed energy transfer}

Clearly, in the case where the donor and acceptor transitions are dipole-allowed, any applied field does not play a measurable role and the transfer 
rate is effectively the field-free result. For the fixed mutual orientation pair we have

$$
\begin{aligned}
\Gamma^{\text {pair }}=\Gamma= & \left(2 \pi \rho_{\mathrm{f}} / \hbar\right) \mu_{i}^{\times 0} \bar{\mu}_{k}^{\times 0} \mu_{j}^{\beta 0} \bar{\mu}_{j}^{\beta 0} \\
& \times \boldsymbol{V}_{(i j)}(K, \boldsymbol{R}) \overline{\boldsymbol{V}}_{(k l)}(K, \boldsymbol{R}) .
\end{aligned}
$$

The rate for two free molecules is obtained after two second-rank rotational averages:

$$
\begin{aligned}
\Gamma^{\text {free }} & =\langle\Gamma 》 \\
& =\left(2 \pi \rho_{\mathrm{f}} / h\right)(1 / 9)\left|\boldsymbol{\mu}^{\alpha 0}\right|^{2}\left|\boldsymbol{\mu}^{(\beta 0}\right|^{2} A(K, R) .
\end{aligned}
$$

where $A(K, R)$ is the excitation transfer function introduced previously elsewhere [7]:

$$
\begin{aligned}
A(K, R) \equiv & V_{(i j)}(K, R) \bar{V}_{(i j)}(K, R)=\left(16 \pi^{2} \varepsilon_{0}^{2} R^{6}\right)^{-1} \\
& \times 2\left(3+K^{2} R^{2}+K^{4} R^{4}\right) .
\end{aligned}
$$

These are well-known standard results [8].

\subsubsection{Electric-field-induced energy transfer}

The rotating pair rate of electric-field-induced energy transfer, corresponding to the case where either the donor or acceptor transition is electricdipole-forbidden, is evaluated with a second rank rotational average and gives

$$
\begin{aligned}
\Gamma^{\text {pair }}= & \langle\Gamma\rangle=\left(2 \pi \rho_{\mathrm{f}} / \hbar\right)\left(D / \varepsilon_{0}\right)^{2} \\
& \times(1 / 3)\left\{S_{i j}^{\alpha 0} \bar{S}_{i m}^{\alpha 0} \mu k \bar{\mu}_{n}^{\beta 0} V_{(j k)}(K, \boldsymbol{R}) \bar{V}_{(m n)}(K, \boldsymbol{R})\right. \\
& +(\mathrm{A} \leftrightarrow \mathrm{B})+2 \operatorname{Re}\left[S_{i j}^{\alpha 0} \bar{\mu}_{n}^{\alpha 0} \bar{S}_{i m}^{\beta 0} \mu k_{k}^{\beta 0}\right. \\
& \left.\left.\times V_{(j k)}(K, \boldsymbol{R}) \overline{\boldsymbol{V}}_{(m) !}(K, \boldsymbol{R})\right]\right\}
\end{aligned}
$$

Two further averages (rank two for the diagonals, three for the interference term) produce the free molecule rate

$$
\begin{aligned}
\Gamma^{\mathrm{frec}} & =\langle\langle\langle\Gamma\rangle\rangle\rangle=\left(2 \pi \rho_{\mathrm{f}} / \hbar\right)\left(D / \varepsilon_{0}\right)^{2} \\
& \times(1 / 54)\left\{2 S_{i j}^{\alpha 0} \bar{S}_{i j}^{\alpha 0}\left|\boldsymbol{\mu}^{\beta 0}\right|^{2} A(K, \boldsymbol{R})+(\mathrm{A} \leftrightarrow \mathrm{B})\right. \\
& +\varepsilon_{i j k} S_{i j}^{\alpha 0} \bar{\mu}_{k}^{\alpha 0} \varepsilon_{l m n} \bar{S}_{l m}^{\beta 0} \mu_{n}^{\beta 0} \\
& \left.\times\left(A(K, R)-A^{\prime}(K, R)\right)\right\}
\end{aligned}
$$

where we introduce another energy transfer function that is only of considerable magnitude at large intermolecular distances $[16,20]$ :

$$
\begin{aligned}
A^{\prime}(K, R) & \equiv V_{(i i)}(K, R) \bar{V}_{(j j)}(K, R) \\
& =\left(16 \pi^{2} \varepsilon_{0}^{2} R^{6}\right)^{-1} \times 4 K^{4} R^{4} .
\end{aligned}
$$

Generally not all terms of eq. (23) will actually contribute. If for example the donor transition $x \rightarrow 0$ is dipole-forbidden, only the first diagonal term of the rate equation remains

\subsection{Polar molecules}

We now consider the general case where both donor and acceptor species have finite permanent dipole moments; the case when just one species is polar is thereby also included.

\subsubsection{Dipole-allowed energy transfer}

Since in the rotating pair case both molecules are in a fixed mutual orientation, the electric field can only influence the entity A $\cdots \mathrm{B}$; however, this will not influence the energy transfer in any way, so the rate is again

$$
\begin{aligned}
\Gamma^{\mathrm{pair}}=\Gamma= & \left(2 \pi \rho_{\mathrm{f}} / \hbar\right) \mu_{i}^{\chi 0} \bar{\mu}_{k}^{\chi 0} \mu_{j}^{\beta 0} \bar{\mu} l^{\beta 0} \\
& \times V_{(i j)}(K, \boldsymbol{R}) \bar{V}_{(k l)}(K, \boldsymbol{R}) .
\end{aligned}
$$

The situation changes for free molecules because now each molecule with its static dipole moment ${ }^{\mathrm{A}} \boldsymbol{\mu}^{00}$ (or ${ }^{\mathrm{B}} \boldsymbol{\mu}^{00}$ ) experiences a torque of magnitude $\varepsilon_{0}^{\prime}\left(\boldsymbol{\mu}^{00} \times \boldsymbol{D}\right)$ exerted by the field. As a consequence the orientational distribution is no longer isotropic. The rotational averaging procedure is necessarily different [21], and must accommodate Boltzmann weighting factors corresponding to the orientating influence of the electric field;

$\exp \left[{ }^{\mathrm{A}} \boldsymbol{\mu}^{00} \cdot\left(\boldsymbol{D} / \varepsilon_{0}\right) /\left(k_{\mathrm{B}} T\right)\right], \quad \exp \left[{ }^{\mathrm{B}} \boldsymbol{\mu}^{00} \cdot\left(\boldsymbol{D} / \varepsilon_{0}\right) /\left(k_{\mathrm{B}} T\right)\right]$.

First, the frame of molecule $A$ has to be decoupled from the frames of B, R and $D$; second, $B$ has to be decoupled from $\boldsymbol{R}$ and $\boldsymbol{D}$. The averaged rates are:

$$
\begin{aligned}
\langle\Gamma\rangle= & \left\langle\Gamma \times \exp \left[{ }^{\mathrm{A}} \boldsymbol{\mu}^{00} \cdot\left(\boldsymbol{D} / \varepsilon_{0}\right) /\left(k_{\mathrm{B}} T\right)\right]\right\rangle / \\
& \left\langle\exp \left[{ }^{\mathrm{A}} \boldsymbol{\mu}^{00} \cdot\left(\boldsymbol{D} / \varepsilon_{0}\right) /\left(k_{\mathrm{B}} T\right)\right]\right\rangle, \\
\langle\Gamma\rangle= & \left\langle\langle\Gamma\rangle \times \exp \left[{ }^{\mathrm{B}} \boldsymbol{\mu}^{00} \cdot\left(\boldsymbol{D} / \varepsilon_{0}\right) /\left(k_{\mathrm{B}} T\right)\right]\right\rangle / \\
& \left\langle\exp \left[{ }^{\mathrm{B}} \boldsymbol{\mu}^{00} \cdot\left(\boldsymbol{D} / \varepsilon_{0}\right) /\left(k_{\mathrm{B}} T\right)\right]\right\rangle .
\end{aligned}
$$

The calculation procedure is described in detail in ref. [21] and outlined in ref. [12]; it gives rise to spherical Bessel functions $j_{n}\left(-i \gamma_{\mathrm{A}}\right)$ and $j_{n}\left(-i \gamma_{\mathrm{B}}\right)$ 


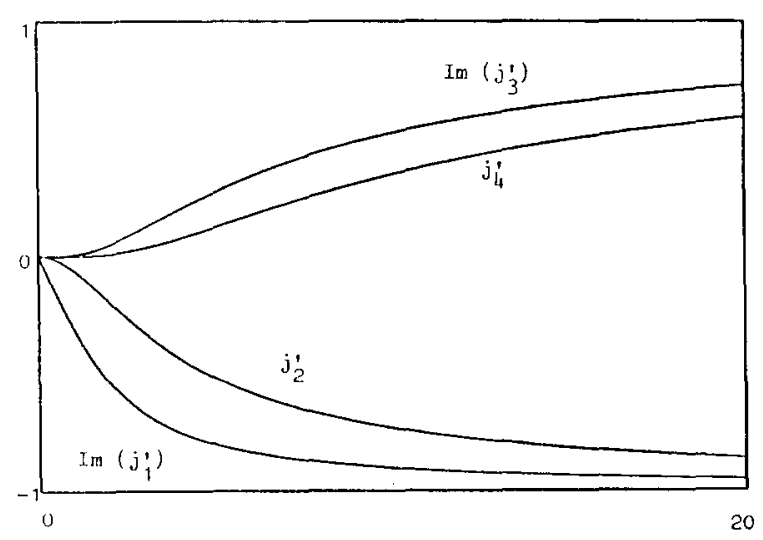

Fig. 4. Plot of the reduced spherical Bessel functions $j_{n}^{\prime}(-i \gamma)$ versus the parameter $\gamma=\mu^{00}\left(D / \varepsilon_{0}\right) /\left(k_{\mathrm{B}} T\right)$. Note that for even $n$ the values are real; for the purely imaginary odd $n$ functions, $\operatorname{Im}\left[j_{1}^{\prime}(-i \gamma)\right]$ and $\operatorname{Im}\left[j_{3}^{\prime}(-i \gamma)\right]$ are plotted. The abscissa scale extends from $\gamma=0$ to 20 ; with $\mu^{00}=5 \times 10^{-30} \mathrm{Cm}$ and $T=300 \mathrm{~K}$ this corresponds to field strengths $0 \leqslant\left(D / \varepsilon_{0}\right) \leqslant 1.6 \times 10^{10} \mathrm{Vm}^{-1}$.

$(n=1,2,3,4)$ whose imaginary arguments have a modulus given by

$\gamma_{\mathrm{A}} \equiv{ }^{\mathrm{A}} \mu^{00}\left(D / \varepsilon_{0}\right) /\left(k_{\mathrm{B}} T\right)$,

$\gamma_{\mathrm{B}} \equiv{ }^{\mathbf{B}} \mu^{00}\left(D / \varepsilon_{0}\right) /\left(k_{\mathrm{B}} T\right)$.

By virtue of the calculation the unit vectors $\hat{\boldsymbol{D}},{ }^{\mathrm{A}} \hat{\boldsymbol{\mu}}^{00}$ and ${ }^{\mathbf{B}} \hat{\boldsymbol{\mu}}^{00}$ appear; the latter two may be defined as the $z$-axes of the donor and acceptor. Following evaluation of the first two averages by eqs. (27) and (28) the third average with respect to the field must be carried out: this is a conventional non-weighted average over the mutual orientations of $\boldsymbol{D}$ and $\boldsymbol{R}$. In passing it may be noted that although the ordering of the first two rotational averages is arbitrary, the field average must be performed last. The overall result is then:

$$
\begin{aligned}
\Gamma^{\text {free }}= & \langle\langle\langle\Gamma\rangle\rangle\rangle \\
= & \left(2 \pi \rho_{\mathrm{f}} / \hbar\right)(1 / 180)\left\{20\left|\boldsymbol{\mu}^{\alpha 0}\right|^{2}\left|\boldsymbol{\mu}^{\beta 0}\right|^{2} A(K, R)\right. \\
& +{ }^{{ }^{\prime} j_{2}^{\prime} j_{2}^{\prime}}\left(3\left(\mu_{z}^{\alpha 0}\right)^{2}-\left|\mu^{\alpha 0}\right|^{2}\right)\left(3\left(\mu_{z}^{\beta 0}\right)^{2}\right. \\
& \left.\left.-\left|\mu^{\beta 0}\right|^{2}\right)\left(A(K, R)+3 A^{\prime}(K, R)\right)\right\},
\end{aligned}
$$

where the $j_{2}^{\prime}$ are reduced spherical Bessel functions defined in general by [12]:

$$
\begin{aligned}
& \mathrm{A}_{j_{n}^{\prime}} \equiv j_{n}\left(-\mathrm{i} \gamma_{\mathrm{A}}\right) / j_{0}\left(-\mathrm{i} \gamma_{\mathrm{A}}\right), \\
& \mathbf{B}_{n}^{\prime} \equiv j_{n}\left(-\mathrm{i} \gamma_{\mathrm{B}}\right) / j_{0}\left(-\mathrm{i} \gamma_{\mathrm{B}}\right) .
\end{aligned}
$$

Reduced spherical Bessel functions with $n=1,2,3$ and 4 feature in the results of later calculations; fig. 4 depicts how each depends on its argument.

The first point to make about the result (31) is that its first part equals the (field-free) result for an isotropic system, eq. (20). In the light of this remark, the particular process under discussion may be envisaged as electric field-modified energy transfer; the magnitude of the anisotropy correction is discussed in section 5. It is perhaps worth drawing attention to the fact that if either (donor or acceptor) static moment is zero, all the reduced spherical Bessel functions $j_{n}^{\prime}(n \geqslant 1)$ become zero, too, and the rate equation completely reduces to the isotropic result. Clearly the electric field modifies the rate only if both molecules are polar.

\subsubsection{Electric-field-induced energy transfer}

Finally we address the most complex case in which energy transfer is not only induced by application of the static field, but is also influenced by a degree of molecular alignment which the field confers upon the system.

For the rotating pair an overall static dipole moment $\mu^{00}$ exists, defined by the vector sum

$\mu^{00} \equiv{ }^{A} \mu^{00}+{ }^{B} \mu^{00}$

and the Boltzmann weighting factor is

$\exp \left[\boldsymbol{\mu}^{00} \cdot\left(\boldsymbol{D} / \varepsilon_{0}\right) /\left(k_{\mathrm{B}} T\right)\right]$.

In analogy to eqs. (29), (30), (32) and (33) we can define

$\gamma \equiv \mu^{00}\left(D / \varepsilon_{0}\right) /\left(k_{\mathrm{B}} T\right), \quad j_{n}^{\prime} \equiv j_{n}(-\mathrm{i} \gamma) / j_{0}(-\mathrm{i} \gamma)$,

Calculation of the appropriate second rank weighted rotational average gives the pair result:

$$
\begin{aligned}
\langle\Gamma\rangle= & \left(2 \pi \rho_{\mathrm{f}} / \hbar\right)\left(D / \varepsilon_{0}\right)^{2} \\
& \times(1 / 3)\left\{\left[\left(1+j_{2}^{\prime}\right) S_{i j}^{\alpha 0} \bar{S}_{i m}^{\alpha 0}\right.\right. \\
& \left.-3 j_{2}^{\prime} S_{i j}^{\alpha 0} \hat{\mu}_{i}^{00} S_{l m}^{\alpha 0} \hat{\mu}_{l}^{00}\right] \\
& \times \mu_{k}^{\beta 0} \bar{\mu}_{n}^{\beta 0} V_{(j k)}(K, R) \bar{V}_{(m n)}(K, R)+(\mathrm{A} \leftrightarrow \mathrm{B}) \\
& +2 \operatorname{Re}\left(\left[\left(1+j_{2}^{\prime}\right) S_{i j}^{\alpha 0} \bar{S}_{i m}^{\beta 0}\right.\right. \\
& \left.-3 j_{2}^{\prime} S_{i j}^{\alpha 0} \hat{\mu}_{i}^{00} \bar{S}_{l m}^{\beta 0} \hat{\mu}_{l}^{00}\right] \\
& \left.\left.\times \mu_{k}^{\beta 0} \bar{\mu}_{n}^{\alpha 0} V_{(j k)}(K, R) \bar{V}_{(m n)}(K, R)\right)\right\} .
\end{aligned}
$$


If $\boldsymbol{\mu}^{00}$ equals zero (e.g. if the individual moments of the donor and acceptor have an antiparallel alignment), the parameter $j_{2}^{\prime}$ equals zero, and naturally eq. (22) results.

In order to obtain the free molecule rate, one has to consider the orientating effect of the field on the individual static dipoles, and proceed via the three averages as explained in section 3.2.1. The resulting formula for $\Gamma^{\text {free }}$, which involves spherical Bessel functions of each order from $j_{1}^{\prime}$ to $j_{4}^{\prime}$, is very cumbersome and is therefore reserved for an appendix. However, a special case of the result arises if one of the static electric dipole moments, say ${ }^{\mathrm{B}} \mu^{00}$, is zero. Here the following appreciably simpler expression can be shown to apply:

$$
\begin{aligned}
\Gamma^{\text {free }}= & \langle\langle\langle\Gamma\rangle\rangle\rangle=\left(2 \pi \rho_{\mathrm{f}} / \hbar\right)\left(D / \varepsilon_{0}\right)^{2} \times(1 / 2700) \\
& \times\left\{1 0 0 \left[\left(1+{ }^{\mathrm{A}} j_{2}^{\prime}\right) S_{i j}^{\alpha 0} \bar{S}_{i j}^{\alpha 0}\right.\right. \\
& \left.-3^{\mathrm{A}} j_{2}^{\prime} S_{z i}^{\alpha 0} S_{z i}^{\alpha 0}\right]\left|\mu^{\beta 0}\right|^{2} A(K, R) \\
& +100\left|\mu^{\alpha 0}\right|^{2} S_{i j}^{\beta 0} \bar{S}_{i j}^{\beta 0} A(K, R) \\
& +{ }^{\mathrm{A}} j_{2}^{\prime}\left[-2 S_{i j}^{\beta 0} \bar{S}_{i j}^{\beta 0}+3\left(S_{i i}^{\beta 0} \bar{S}_{j j}^{\beta 0}+S_{j i}^{\beta 0} \bar{S}_{j i}^{\beta 0}\right)\right] \\
& \times\left(\left|\mu^{\alpha 0}\right|^{2}-3\left|\mu_{z}^{\alpha 0}\right|^{2}\right)\left[A(K, R)+3 A^{\prime}(K, R)\right] \\
& +50\left[\left(1-2^{\mathrm{A}} j_{2}^{\prime}\right) \varepsilon_{i j k} S_{i j}^{\alpha 0} \bar{\mu}_{k}^{\alpha 0}\right. \\
& \left.+3^{\mathrm{A}} j_{2}^{\prime} \varepsilon_{i j z}\left(S_{i j}^{\alpha 0} \bar{\mu}_{z}^{\alpha 0}-S_{i z}^{\alpha 0} \bar{\mu}_{j}^{\alpha 0}\right)\right] \\
& \left.\times \varepsilon_{l m n} \bar{S}_{l m}^{\beta 0} \mu_{n}^{\beta 0}\left(A(K, R)-A^{\prime}(K, R)\right)\right\} .
\end{aligned}
$$

As in eq. (31) the static dipoles of molecules $A$ and $B$ define the individual $z$-axes. As mentioned earlier, either the acceptor or the donor transition should be forbidden in order to exclude the otherwise dominating dipole-allowed energy transfer. If the acceptor in addition to being non-polar has a dipole-forbidden transition $\left({ }^{\mathrm{B}} \boldsymbol{\mu}^{00}=0, \boldsymbol{\mu}^{\beta \mathrm{O}}=0\right)$, then only the second term contributes; if the donor transition $\alpha \rightarrow 0$ is forbidden $\left(\boldsymbol{\mu}^{\alpha 0}=0\right)$, only the first term survives. Note that in eq. (38) the orienting effect of the field is already manifest in terms linear in the reduced spherical Bessel functions, whereas in the dipoleallowed case (31) the effect depends on them quadratically.

\section{Selection rules}

As established earlier, the selection rules for dipole-allowed energy transfer entail the normal selection rule for one-photon emission by the donor and one-photon absorption by the acceptor. Electric-field-induced energy transfer usually features a two-quantum (Raman-like) selection rule for one, and a one-quantum selection rule for the other molecule.

These rules can be understood from the timeordered diagrams (figs. 1 and 2). In fig. 1 there are just two electric interactions between the molecules and the (radiation) field, corresponding to the only permissible two-fold partition of 2 which is $(1,1)$. In fig. 2 we have three interactions, giving the partitions $(2,1)$ and $(1,2)$, i.e. interactions which invoke the two-quantum selection rule occur either at the donor $A$ or the acceptor B, cf. ref. [22].

For a detailed analysis one can employ irreducible tensors to elucidate the selection rules associated with the transition dipole moments $\boldsymbol{\mu}^{\alpha 0}, \boldsymbol{\mu}^{\beta 0}$ and the $S^{x 0}$ and $S^{\beta 0}$ tensors. Any such molecular transition tensor is characterised by two types of parameter, the parity and the weights of which the tensor is comprised [23]. The parity of a molecular transition equals $(-1)^{P}$ for $p$ dipolar interactions between the molecule and an electric field (static or radiation); e.g. for the partition $(2,1)$ we obtain positive parity for molecule $\mathrm{A}\left(S^{\times 0}\right)$ and negative parity for $\mathrm{B}\left(\mu^{\beta 0}\right)$. Naturally if $|0\rangle$ signifies a totally symmetric ground state, these parity labels may be identified with the symmetries of the excited states $\alpha$ and $\beta$, respectively.

Tensor weights can be interpreted as relating to the modulus of a change in electronic angular momentum, $|\Delta L|$. Any tensor $T$ can be decomposed into a sum of irreducible tensors $T^{(0)}+T^{(1)}+$ $T^{(2)}+\ldots$, the latter distinguished by these weights $w=0,1,2, \ldots$. The dimension or rank of $T$ gives the upper limit for $w\left(1\right.$ for $\boldsymbol{\mu}^{\alpha 0}$ and $\boldsymbol{\mu}^{\beta 0}, 2$ for the $S$ tensors); the lower bound on $w$ is 1 for rank one and 0 for any other rank. A transition dipole has weight 1 and parity -1 which we denote by the representation $\mathrm{D}^{(1-)}$. A two-quantum transition possesses weights 0,1 and 2 and parity +1 , giving the representation $\mathrm{D}^{(0+1}+\mathrm{D}^{(1+)}+\mathrm{D}^{(2+)}$. 
For energy transfer to occur, the selection rules for both the downward transition of the donor and the upward transition of the acceptor must be satisfied. This in turn requires that for each molecular transition at least one irreducible tensor must be non-zero. Whether this is the case can be ascertained with the point group representation of various tensor weights; the required information is tabulated in ref. [23] (see also ref. [12]). Any representation spanned by components of $\mathrm{D}^{(1-)}$ thus denotes a dipole-allowed transition; when either $\mathrm{D}^{(0+)}, \mathrm{D}^{(1+)}$, or $\mathrm{D}^{(2+)}$ appear, the transition is two-quantum allowed. Dipole-allowed transitions belong to class 1 which is represented by the symbol $1^{-}$(1) [class parity (weights)]. Two-photon allowed trasitions form class 2 which has six members, $2^{+}(012), 2^{+}(12), 2^{+}(02), 2^{+}(2), 2^{+}(1)$ and $2^{+}(0)$ [23]. Here for example (012) means that weights 0 and 1 and 2 are all spanned by the representation of the transition.

Whenever the representations of both transitions $\alpha \rightarrow 0$ and $0 \rightarrow \beta$ belong to class $1^{-}(1)$, dipole-allowed energy transfer occurs, subject of course to energy conservation. If one of the transitions does not belong to this class, it will very often belong to class 2 . In such circumstances, however, it must be borne in mind that although all class 2 transitions are allowed as electric-field-induced transitions, some are also allowed through a single-quantum electric quadrupole interaction, as the latter entails the irreducible representation $\mathrm{D}^{(2+)}$. (Weights 0 and 1 do not appear since the quadrupole is a symmetric and traceless tensor quantity.) The time-ordered diagrams for the associated dipole-quadrupole mediated transfer are the same as for dipole-dipole transfer (see fig. 1) where no static field is involved. Thus whereas all six members of class 2 allow electric field-induced energy transfer, $2^{+}(012), 2^{+}(12), 2^{+}(02)$ and $2^{+}(2)$ additionally allow dipole-quadrupole transfer. This type of energy transfer depends in the near zone on $R^{-8}$, however, and features only in energy transfer between close neighbours (an experimental example is provided in ref. [5]) whereas electric field-induced transfer shows $R^{-6}$ dependence as in the conventional dipole-dipole transfer. The class $2^{+}(1)$ and $2^{+}(0)$ cases for which energy transfer is dipole-quadrupole forbidden, but allowed by field induction, are comprehensively tabulated in ref. [23].

Finally we consider fig. 3 which corresponds to the partition $(2,2)$ (clearly partitions $(3,1)$ and $(1,3)$ are also possible). Here both transitions must belong to class 2 , which allows for doubly electricfield-induced energy transfer. If both representations of the transitions belong to one of the classes $2^{+}(012), 2^{+}(12), 2^{+}(02)$ or $2^{+}(2)$, quadrupole-quadrupole energy transfer may occur (see for example the experiment in ref. [6]).

\section{Discussion}

The first issue which needs to be addressed in the light of the above theory is the likely magnitude of the effects which we have described. Let us first examine the more common case of dipole-allowed energy transfer (the dipole-dipole mechanism) in free molecules. As shown by eq. (31), the orientation of static dipoles in a static electric field modifies the energy transfer rate (20). The second term of eq. (31) is multiplied by ${ }^{{ }^{A}} j_{2}^{\prime}{ }^{B_{j}^{\prime}}{ }_{2}$ which ranges from 0 (for a vanishing dipole, or in the absence of any static field, where $\gamma_{A}$ or $\gamma_{B}$ equal zero) to 1 (for the case of large dipoles or a high field, where $\gamma_{A}$ and $\gamma_{B}$ acquire high values). Ref. [12] gives the limiting analytical form of these functions, and fig. 4 illustrates their exact dependence on the parameter $\gamma$. The onset of field-orientation effects is characterised by a region in which each $j_{2}^{\prime} \approx-\gamma^{2} / 15$ which, from eqs. (29) and (30), clearly results in a departure from the field-free rate associated with a quartic dependence on the field strength and an inverse fourth power dependence on the absolute temperature $T$.

As the remainder of the expression which multiplies the $j^{\prime}$ functions in the second term of eq. (31) is of comparable magnitude to the first term (except for the rare case where $2 \mu_{z}^{2} \approx \mu_{x}^{2}+\mu_{y}^{2}$ for the transition moments of either molecule), it becomes apparent that a very high field of ca. $10^{10} \mathrm{~V} \mathrm{~m}^{-1}$ would substantially modify the rate of energy transfer, whereas a low field would change the rate only marginally. Taking into account the dielectric influence on the local fields experienced in real liquids, values of $D / \varepsilon_{0}$ in excess of $10^{9} \mathrm{~V} \mathrm{~m}^{-1}$ are certainly attainable, and the associated change in energy 
transfer rate should be measurable. The change in rate can be positive or negative, depending on the magnitude and direction of the donor and acceptor transition moments. The high field regime is associated with a rate modification which increases only in inverse proportion to the field strength [12].

A special case arises where A and B are chemically equivalent and $\alpha=\beta$, as is frequently the case. Here the modification to the rate invariably carries a positive sign. Consequently the effect of applying the field is to enhance the energy transfer process. This can be understood as a direct result of the action of the field in creating a preferential alignment of the acceptor with respect to the donor species. In the optimum case where the transition dipole lies in the $z$-direction (i.e. it is parallel to the permanent electric moment), and assuming that the transfer process is dominated by donor-acceptor pairs lying in relatively close proximity (i.e. the near zone, where $K R \ll 1$ ), then the rate is enhanced by a factor of $0.2\left({ }^{A} j_{2}^{\prime}\right)^{2}$, as follows from eq. (31). The maximum degree of enhancement occurs at high fields where the reduced spherical Bessel function tends to unity, as shown in fig. 4 , leading to a $20 \%$ rate increase. This behaviour ought to be readily amenable to experimental confirmation.

For the remainder of this discussion we turn our attention to electric-field-induced energy transfer. It is appropriate to recast the salient rate equation in a form which accommodates the band structure of the donor emission and the acceptor absorption spectra, as in the standard Förster theory. The procedure follows along similar lines to those recently discussed in detail elsewhere $[8,15]$. The simplest case arises when the static dipoles are zero and when only the first term in the rate equation (17), eq. (23) is present (i.e. $\boldsymbol{\mu}^{\alpha 0}=0$ ). Then the following energy transfer rate applies for fluid samples:

$\Gamma^{\text {free }}=9\left(8 \pi \tau_{\mathrm{A}}\right)^{-1} \int_{0}^{\infty} F_{\mathrm{A}}^{\prime}(\omega) \sigma_{\mathrm{B}}(\omega) K^{2} g(K, R) \mathrm{d} \omega$,

where

$$
\begin{aligned}
g(K, R) \equiv & {\left[6(K R)^{-6}+2(K R)^{-4}\right.} \\
& \left.+2(K R)^{-2)}\right] / 9,
\end{aligned}
$$

$$
\begin{aligned}
\sigma_{\mathbf{B}}(\omega) \equiv & \pi \omega\left|\boldsymbol{\mu}^{\beta 0}\right|^{2}\left(3 \varepsilon_{0} c\right)^{-1} \rho_{\mathrm{f}}\left(E_{\beta 0}-\hbar \omega\right), \\
F_{\mathbf{A}}^{\prime}(\omega) \equiv & \omega^{3} \tau_{\mathbf{A}} S_{i j}^{\alpha 0} \bar{S}_{i j}^{\alpha 0} D^{2} /\left(27 \varepsilon_{0}^{2}\right)\left(3 \varepsilon_{0} \pi c^{3}\right)^{-1} \\
& \times \rho_{\mathrm{f}}\left(E_{\alpha 0}-\hbar(\omega) .\right.
\end{aligned}
$$

Here $\sigma_{\mathrm{B}}(\omega)$ denotes the absorption cross-section of the acceptor molecule $\mathrm{B}$ and $F_{\mathrm{A}}^{\prime}(\omega)$ designates the electric-field-induced emission spectrum of the donor A.

Cast in the above form (39), the essential similarity of the rate equation to that which applies to conventional dipole-allowed transfer can be seen [8]; for that process, the rate is as given by eq. (39) but with $F_{\mathrm{A}}^{\prime}(\omega)$ replaced by $F_{\mathrm{A}}(\omega)$, where

$F_{\mathrm{A}}(\omega) \equiv \omega^{3} \tau_{\mathrm{A}}\left|\boldsymbol{\mu}^{\alpha 0}\right|^{2}\left(3 \varepsilon_{0} \pi c^{3}\right)^{-1} \rho_{\mathrm{f}}\left(E_{\alpha 0}-\hbar \omega\right)$,

which entails replacing the term $S_{i j}^{\alpha 0} \bar{S}_{i j}^{\alpha 0} D^{2} /\left(27 \varepsilon_{0}^{2}\right)$ by $\left|\boldsymbol{\mu}^{\alpha 0}\right|^{2}$. The definition (16) of the $S$ tensor shows that $S_{i j}^{\alpha 0} \bar{S}_{i j}^{\alpha 0}$ is of the same order of magnitude as $\left|\mu^{\alpha 0}\right|^{4} / \Delta E^{2}$, where $\Delta E$ is a typical molecular energy gap, e.g. $3 \times 10^{-20} \mathrm{~J}$. Assuming that $\left|\boldsymbol{\mu}^{\alpha 0}\right|$ is in the range of $5 \times 10^{-30} \mathrm{C} \mathrm{m}$, the rate of induced transfer would equal that of dipole-allowed transfer for a field of $D / \varepsilon_{0} \approx 3 \times 10^{10} \mathrm{~V} \mathrm{~m}^{-1}$; nonetheless for a routinely applied field of ca. $10^{8} \mathrm{~V} \mathrm{~m}^{-1}$ the electric-field-induced transfer rate should still be readily measurable. In passing we note that fields of that magnitude can arise on the microscopic scale through localised ion field effects, not necessarily through application of a macroscopic field. Similar remarks can be made concerning the case of a rotating pair or for molecules with a fixed orientation in the static field.

The most interesting feature of the field-induced process lies in the possibility of inducing a donor decay process which is otherwise forbidden if there is no applied field. Suppose that molecules of the species $\mathrm{A}$ are excited to a metastable state, for example by laser excitation to some higher vibronic level and subsequent decay to the state $|\alpha\rangle$. One could then store energy in the sample over short periods, up to times approaching the lifetimes of internal decay mechanisms. In fact the successive application of an electric field has already been considered as a means of inducing spontaneous emission $\alpha \rightarrow 0$ (e.g. in $\mathrm{H}_{2}$ or $\mathrm{N}_{2}$ ), providing the basis for construction of a molecular switch for energy "dumping" [24]. However if the excited 
molecules A were surrounded by suitable acceptors B, for example a laser dye, the process after switching on the field might be energy transfer rather than emission, possibly forming the basis for a novel kind of laser system.

\section{Appendix}

The general expression for the rate of electric field-induced energy transfer between free molecules is

$$
\begin{aligned}
& \Gamma^{\text {free }}=\langle\langle\langle\Gamma\rangle\rangle\rangle=\left(2 \pi \rho_{\mathrm{f}} / h\right)\left(D / \varepsilon_{0}\right)^{2}(1 / 94500)
\end{aligned}
$$

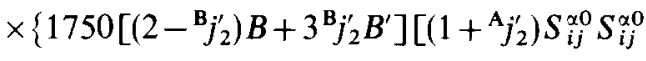

$$
\begin{aligned}
& \left.-{ }^{\mathrm{A}_{j}^{\prime}} S_{z i}^{\alpha 0} S_{z i}^{\alpha 0}\right] A(K, R) \\
& +{ }^{\mathrm{B}_{j}^{\prime}}{ }_{2}\left(B-3 B^{\prime}\right)\left\{105\left[S_{i i}^{\alpha 0} S_{j j}^{\alpha 0}+S_{i j}^{\alpha 0} S_{i j}^{\alpha 0}\right)\right. \\
& \times\left(A(K, R)+3 A^{\prime}(K, R)\right) \\
& \left.+2 S_{i j}^{\alpha 0} S_{i j}^{\alpha 0}\left(8 A(K, R)-A^{\prime}(K, R)\right)\right] \\
& +75^{\mathrm{A}} j_{2}^{\prime}\left[\left(-6 S_{i i}^{\alpha 0} S_{z z}^{\alpha 0}\right.\right. \\
& -3 S_{i z}^{\alpha 0} S_{i z}^{\alpha 0}-6 S_{i z}^{\alpha 0} S_{z i}^{\alpha 0}+2 S_{i i}^{\alpha 0} S_{j j}^{\alpha 0}+S_{i j}^{\alpha 0} S_{i j}^{\alpha 0} \\
& \left.+2 S_{i j}^{\alpha 0} S_{j i}^{\alpha 0}\right)\left(A(K, R)+3 A^{\prime}(K, R)\right)+\left(S_{i j}^{\alpha 0} S_{i j}^{\alpha 0}\right. \\
& \left.\left.-3 S_{z i}^{\alpha 0} S_{z i}^{\alpha 0}\right)\left(11 A(K, R)-4 A^{\prime}(K, R)\right)\right] \\
& +45^{A} j_{4}^{\prime}\left[-35 S_{z z}^{\alpha 0} S_{z z}^{\alpha 0}+10 S_{i i}^{\alpha 0} S_{z z}^{\alpha 0}\right. \\
& +5 S_{i z}^{\alpha 0} S_{i z}^{\alpha 0}+10 S_{i z}^{\alpha 0} S_{z i}^{\alpha 0}-5 S_{z i}^{\alpha 0} S_{z i}^{\alpha 0}-S_{i j}^{\alpha 0} S_{i j}^{\alpha 0} \\
& \left.\left.-S_{i i}^{\alpha 0} S_{j i}^{\alpha 0}-S_{i j}^{\alpha 0} S_{j i}^{\alpha 0}\right]\left(A(K, R)-3 A^{\prime}(K, R)\right)\right\} \\
& +(\mathrm{A} \leftrightarrow \mathrm{B})+1750\left[\varepsilon_{i j k} S_{i j}^{\alpha 0} \mu_{k}^{\alpha 0}\left(1-{ }^{\mathrm{A}} j_{2}^{\prime}\right)\right. \\
& \left.+3^{\mathrm{A}} j_{2}^{\prime} \varepsilon_{i j z}\left(S_{i j}^{\alpha 0} \mu_{z}^{\alpha 0}-S_{i z}^{\alpha 0} \mu_{j}^{\alpha 0}\right)\right] \\
& \times\left[\varepsilon_{l m n} S_{l m}^{\beta 0} \mu_{n}^{\beta 0}\left(1-{ }^{\mathbf{B}_{2}^{\prime}}\right)+3^{\mathbf{B}_{j_{2}^{\prime}} \varepsilon_{l m z}}\left(S_{l m}^{\beta 0} \mu_{z}^{\beta 0}\right.\right. \\
& \left.\left.-S_{l z}^{\beta 0} \mu_{m}^{\beta 0}\right)\right]\left(A(K, R)-A^{\prime}(K, R)\right) \\
& +126\left[3 ( { } ^ { \mathrm { A } } j _ { 1 } ^ { \prime } + { } ^ { \mathrm { A } } j _ { 3 } ^ { \prime } ) \left(S_{i i}^{\alpha 0} \mu_{z}^{\alpha 0}\right.\right. \\
& \left.+S_{i z}^{\alpha 0} \mu_{i}^{\alpha 0}\right)+\left(-2^{\mathrm{A}}{j_{1}^{\prime}}_{1}+{ }^{\mathrm{A}_{j}^{\prime}}{ }_{3}\right) S_{z i}^{\alpha 0} \mu_{i}^{\alpha 0} \\
& \left.-15^{\mathrm{A}} j_{3}^{\prime} S_{z z}^{\alpha 0} \mu_{z}^{\alpha 0}\right]\left[\left[( { } ^ { \mathrm { B } } j _ { 1 } ^ { \prime } + { } ^ { \mathrm { B } } j _ { 3 } ^ { \prime } ) \left(S_{l l}^{\beta 0} \mu_{z}^{\beta 0}\right.\right.\right.
\end{aligned}
$$

$$
\begin{aligned}
& \left.+S_{l z}^{\beta 0} \mu_{l}^{\beta 0}\right)+{ }^{\mathrm{B}} j_{3}^{\prime}\left(S_{z l}^{\beta 0} \mu_{l}^{\beta 0}\right. \\
& \left.\left.-5 S_{z z}^{\beta 0} \mu_{z}^{\beta 0}\right)\right]\left(A(K, R)-3 A^{\prime}(K, R)\right) \\
& \left.-2^{\mathrm{B}} j_{1}^{\prime} S_{z l}^{\beta 0} \mu_{l}^{\beta 0}\left(8 A(K, R)-A^{\prime}(K, R)\right)\right] \\
& +6300\left[\left({ }^{\mathrm{A}} j_{1}^{\prime}+{ }^{\mathrm{A}} j_{3}^{\prime}\right)\left(S_{i i}^{\alpha 0} \mu_{z}^{\alpha 0}+S_{i z}^{\alpha 0} \mu_{i}^{\alpha 0}\right)\right. \\
& +\left(-4^{\mathrm{A}} j_{1}^{\prime}+{ }^{\mathrm{A}} j_{3}^{\prime}\right) S_{z i}^{\alpha 0} \mu_{i}^{\alpha 0} \\
& \left.\left.-5^{\mathrm{A}} j_{3}^{\prime} S_{z z}^{\alpha 0} \mu_{z}^{\alpha 0}\right]^{\mathrm{B}} j_{1}^{\prime} S_{z i}^{\beta 0} \mu_{i}^{\beta 0} A(K, R)\right\},
\end{aligned}
$$

where use has been made of the fact that all tensors are real-valued and the following definitions apply:

$B \equiv\left|\mu^{\beta 0}\right|^{2}$,

$B^{\prime} \equiv\left|\boldsymbol{\mu}^{\beta 0} \cdot{ }^{\mathrm{B}} \hat{\boldsymbol{\mu}}^{00}\right|^{2}=\left|\mu_{z}^{\beta 0}\right|^{2}$.

Again we have assumed that the individual static dipoles are defined as the molecular $z$-axes. From eq. (A.1) we can derive eq. (38) by setting $\boldsymbol{\mu}^{\beta 0}=0$.

\section{References}

[1] J.T. Yardley, Introduction to Molecular Energy Transfer (Academic Press, New York, 1980).

[2] A. Gilbert and J. Baggott, Essentials of Molecular Photochemistry (Blackwell, Oxford, 1991).

[3] S.-T. Levy, M.B. Rubin and S. Speiser, J. Photochem. Photobiol. A 66 (1992) 159.

[4] M. Kaschke, B. Valeur, J. Bourson and N.P. Ernsting, Chem. Phys. Lett. 179 (1991) 544.

[5] J.D. Axe and P.F. Weller, J. Chem. Phys. 40 (1964) 3066.

[6] R.T. Brundage and W.M. Yen, Phys. Rev. B 34 (1986) 8810.

[7] D.L. Andrews, Chem. Phys. 135 (1989) 195.

[8] D.L. Andrews and G. Juzeliūnas, J. Chem. Phys. 96 (1992) 6606.

[9] A.M. Bittner, MSc thesis, University of East Anglia, Norwich (1992)

[10] W.H. Weber and E.A. Cohen, Opt. Lett. 8 (1983) 488.

[11] D.L. Andrews and B.S. Sherborne, Chem. Phys. 88 (1984) 1.

[12] D.L. Andrews and B.S. Sherborne, Chem. Phys. 108 (1986) 357.

[13] D.P. Craig and T. Thirunamachandran, Molecular Quantum Electrodynamics (Academic Press, London, 1984).

[14] E.A. Power and S. Zienau, Philos. Trans. R. Soc. London Ser. A 251 (1959) 427;

R.G. Woolley, Proc. R. Soc. London Ser. A 321 (1971) 557.

[15] D.L. Andrews and G. Juzeliūnas, J. Chem. Phys. 95 (1991) 5513. 
[16] D.L. Andrews and A.M. Bittner, J. Phys. B 26 (1993) 675

[17] E. Hudis, Y. Ben-Aryeh and U.P. Oppenheim, Phys. Rev. A 43 (1991) 3631 .

[18] D.L. Andrews and T. Thirunamachandran, J. Chem. Phys 67 (1977) 5026.

[19] P.E. Schipper, Chem. Phys. 57 (1981) 105.

[20] D.L. Andrews and K.P. Hopkins, Adv. Chem. Phys. 77 (1990) 39.
[21] D.L. Andrews and M.J. Harlow, Phys. Rev. A 29 (1984) 2796.

[22] D.L. Andrews and A.M. Bittner, Chem. Phys. 165(1992) 1.

[23] D.L. Andrews, Spectrochim. Acta 46 A (1990) 871 (in appendix 2 an entry "(1)" is missing for point group $D_{5 h}$. $\mathrm{A}_{2}^{\prime \prime}$, rank $\left.(-1)\right)$.

[24] K. Drühl, M.O. Scully and A.W. Overhauser, Opt. Commun. 38 (1981) 393. 